— BCO-DMO Quick Guide -

BCQ-DMO

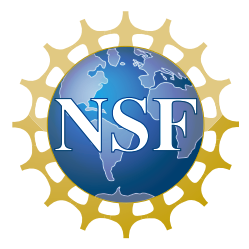




\section{The Data Life Cycle}

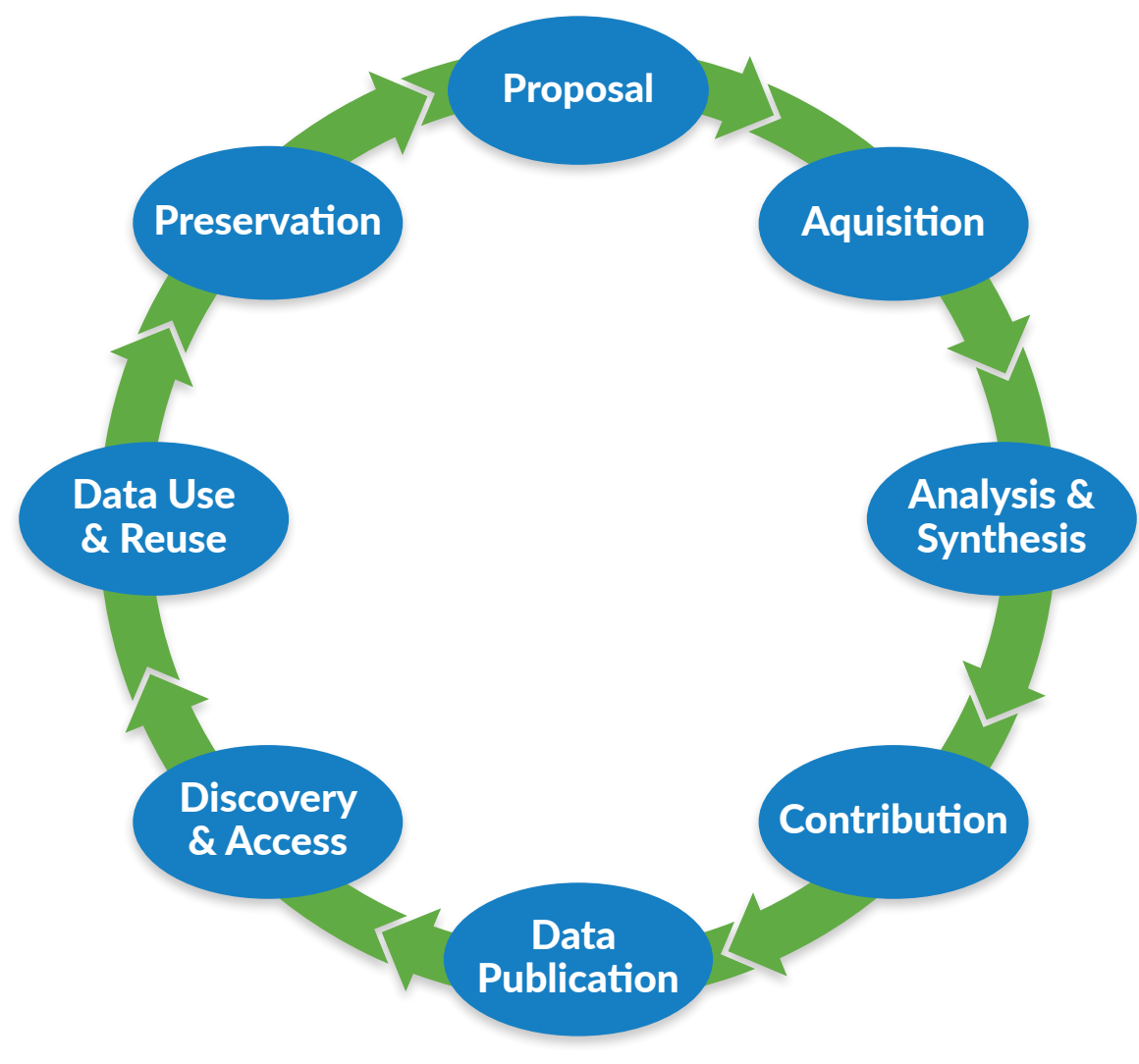

Curating and providing open access to research data is a collaborative process. This process may be thought of as a life cycle with data passing through various phases. Each phase has its own associated actors, roles, and critical activities. Good data management practices are necessary for all phases, from proposal to preservation.

BCO-DMO, a repository funded by the National Science Foundation (NSF), supports the oceanographic research community's data needs throughout the entire data life cycle. This guide describes the services available from BCO-DMO from proposal to preservation and highlights phases where researchers engage significantly with the office.

Data management services are provided free of charge for projects funded via:

- NSF-OCE Biological Oceanography Section

- NSF-OCE Chemical Oceanography Section

- Division of Polar Programs' Antarctic Organisms \& Ecosystems Program

Not funded from the programs above? We can assist in determining the appropriate repository for your project data. See a list of other data management centers ${ }^{1}$. Under certain circumstances, we may negotiate services for data not covered by these NSF sections.
BCO-DMO provides the following services:

- Proposal: Help with your NSF Data Management Plan (see page 2).

- Acquisition: Advice on collecting good metadata and data.

- Contribution: Submission to the database, ensuring compliance with NSF OCE Sample and Data Policy ${ }^{2}$ (NSF 17-037). One-on-one assistance with your data submission (see page 4).

- Data Publication: Datasets are published online at BCO$\mathrm{DMO}^{3}$; citations in just one click; DOIs available (see page 8).

- Discovery \& Access: BCO-DMO search tools (see page 9).

- Data Use \& Reuse: Data are freely accessible*; many types of data are available for new and collaborative research/ modelling/synthesis projects.

- Preservation: BCO-DMO works with the appropriate national data center for long-term archiving (see page 13).

* If you need a limited additional period of time while manuscripts are prepared for publication before your data are publicly available at BCO-DMO, have a conversation with your Program Manager.

https://www.bco-dmo.org/how-get-started\#other_data_center

${ }^{2}$ NSF 17-037; https://nsf.gov/pubs/2017/nsf17037/nsf17037.jpp?org=NSF

${ }^{3}$ https://www.bco-dmo.org 


\section{BCO-DMO Data Management Plan Template}

Proposals submitted to NSF must include a supplementary document of no more than two pages labeled "Data Management Plan” (DMP). This supplementary document should describe how the proposal will conform to NSF's policy on the dissemination and sharing of research results.

Investigators working under awards granted by the NSF Division of Ocean Sciences (OCE) have additional conditions to which they must adhere, as described in the Division of Ocean Sciences Sample and Data Policy ${ }^{1}$.

\section{How BCO-DMO can help}

BCO-DMO has developed a Data Management Plan template to assist investigators in submission of plans that meet the NSF OCE Sample and Data Policy requirements. The template can be found and completed on the DMPTool website.

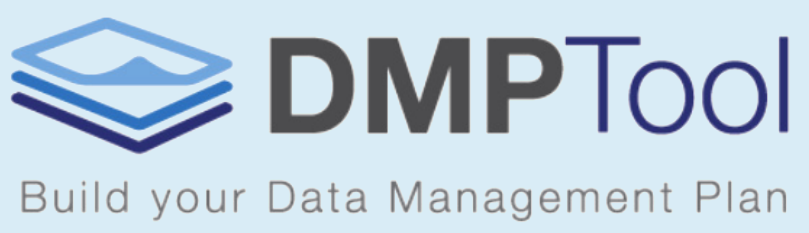

\section{About DMPTool}

$\mathrm{DMPTool}^{2}$ is a free, open-source, online application that helps researchers create data management plans. The DMPTool provides detailed guidance and links to informational resources and walks researchers through the process of generating comprehensive plans tailored to specific DMP requirements, in this case, the NSF OCE requirements.

If you are a researcher from one of the DMPTool partner institutions, you can log in using your institutional credentials. If your institution is not a partner, you can create your own account using any email address. In each section of the DMP template, you will see instructions containing the question or a description of information that should be provided to meet the specific requirement. Each question/requirement also has an example answer and links to additional guidance. The plan may be saved at any point, and can also be shared with collaborators. Once complete, your DMP can be exported in several different formats for inclusion in your NSF proposal.

\section{Additional Resources}

More information on NSF's data management requirements is available on BCO-DMO's website ${ }^{3}$. Detailed instructions on how to use BCO-DMO's DMPTool template are also available in our “Getting Started with DMPTool” guide 4
Image from BCO-DMO's

"Getting Started with DMPTool" guide".

\section{Find the BCO-DMO Template:}

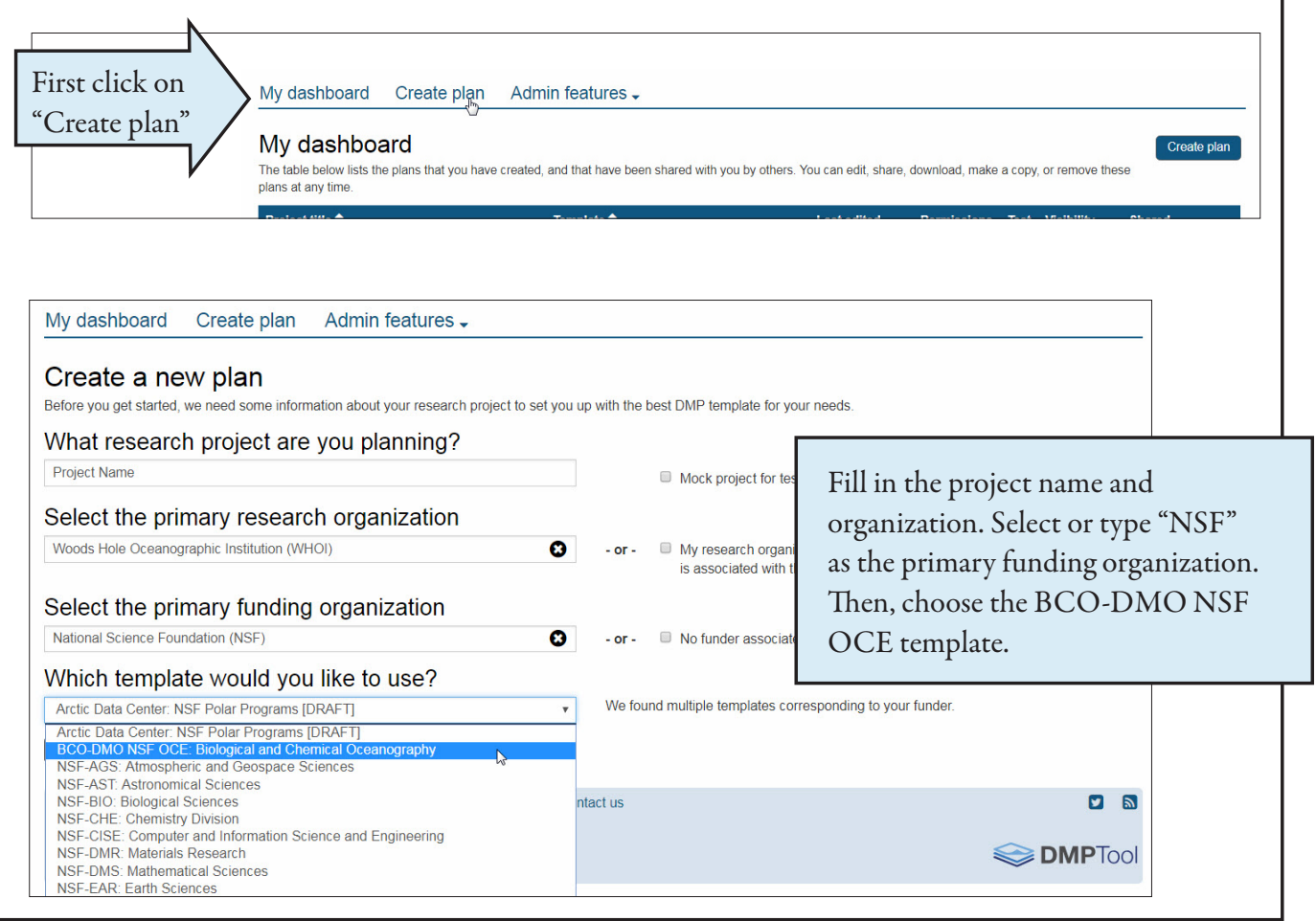

${ }^{1}$ https://www.nsf.gov/pubs/2017/nsf17037/nsf17037.jpp

2https://dmptool.org/

${ }^{3}$ https://www.bco-dmo.org/nsf-two-page-data-management-plan

${ }^{4}$ https://www.bco-dmo.org/sites/default/ les/page_les/BCODMO_Getting_Started_with_DMPTool.pdf 


\section{Contributing Data to BCO-DMO}

By depositing your project information and data into BCO-DMO, your data become shareable, citable resources available for community reuse. We are continually striving to make this process more efficient and streamlined, and welcome your feedback

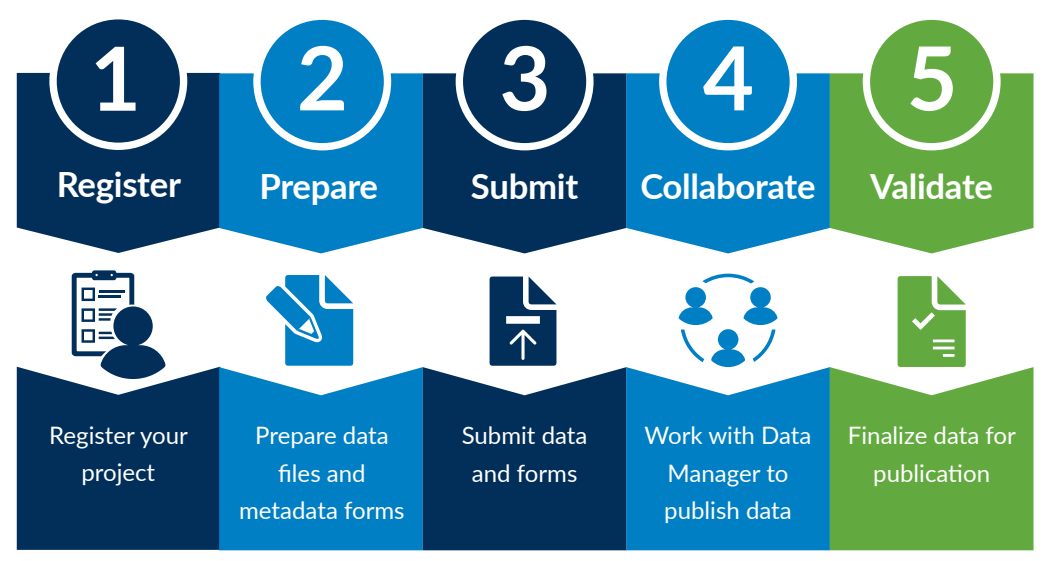

\section{(1) Register a Project}

A. Search BCO-DMO to see if your award is already in our system. If not,

B. Complete a Project Metadata Form ${ }^{1}$ to provide information about projects that are not already registered at BCO-DMO.

- A project oversees a collection of one or more datasets.

- There is usually one project per NSF award with the exception of Collaborative Research awards where one project is funded by multiple award numbers. Some time-series projects may contain multiple awards as well.

- If you do not yet know the NSF award number, please provide as much information as possible including the project title and investigator contact information.

C. Submit your NSF Data Management Plan² with the Project Metadata Form.

\section{Prepare Data and Metadata}

A. Prepare the data files, including error checking and formatting. Understand what is meant by a 'dataset' relative to BCO-DMO (see page 4).

B. Complete a Dataset Metadata Form ${ }^{3}$ to provide information about each unique dataset. See 'Preparing and contributing metadata' (page 4).

C. If data were collected from a research vessel, mooring, glider, or other unique deployment, complete a Deployment Metadata Form ${ }^{4}$.

- Complete this form only if it is applicable to the dataset(s) you are submitting.

- Deployments help describe the geographic and temporal scale of datasets and provide context for mapping the associated data.

- The Rolling Deck to Repository, R2R $\mathrm{R}^{5}$, provides cruise data for vessels in the UNOLS fleet. For data on these cruises, you need only reference the R2R cruise identifier and $\mathrm{BCO}-\mathrm{DMO}$ does the rest.

\section{Submit}

Send applicable metadata forms and data files to info@bco-dmo.org.

- You will receive a reply from one of our Data Managers confirming receipt of your forms and data files, if applicable.

- If a data set is too large to send as an email attachment, please contact us for instructions on the best way to contribute your data.

- NOTE: We strongly encourage you to submit data at least one month in advance of any pressing deadlines (e.g. NSF reports, manuscript publication) to provide adequate data processing time.

\section{Collaborate}

A Data Manager will begin the process of making the data available online. We strive to develop robust metadata that will ensure the data are easily discoverable and reusable. Your Data Manager will contact you with follow-up questions or requests for more information to ensure that the metadata is complete. This may be an iterative process, so your patience and cooperation are greatly appreciated.

\section{Validate}

Once your datasets are online, you'll be asked to review the data and metadata for completeness and accuracy. This validation stage is the final step in the process, and necessary for assignment of DOIs and long-term archive.

Once datasets are reviewed and validated by the contributor, BCODMO ensures that the data are archived properly at the appropriate National Data Center (e.g. National Centers for Environmental Information, $\mathrm{NCEI}^{6}$ ). 


\section{Preparing Data and Metadata}

You should submit data in the format most appropriate for your community. If this format is proprietary or non-tabular, Data Managers will create a tabular version of your data to import into the BCO-DMO data system. If the most appropriate format is not one of the various output formats provided by the BCO-DMO data system (e.g. .csv., .tsv, .nc, .mat), we will work with you to arrive at the best data representation possible.

\section{Data before and after submission}

\section{Before}

These are plain text files of the same type (e.g. one file per cast) so they can be combined into one dataset

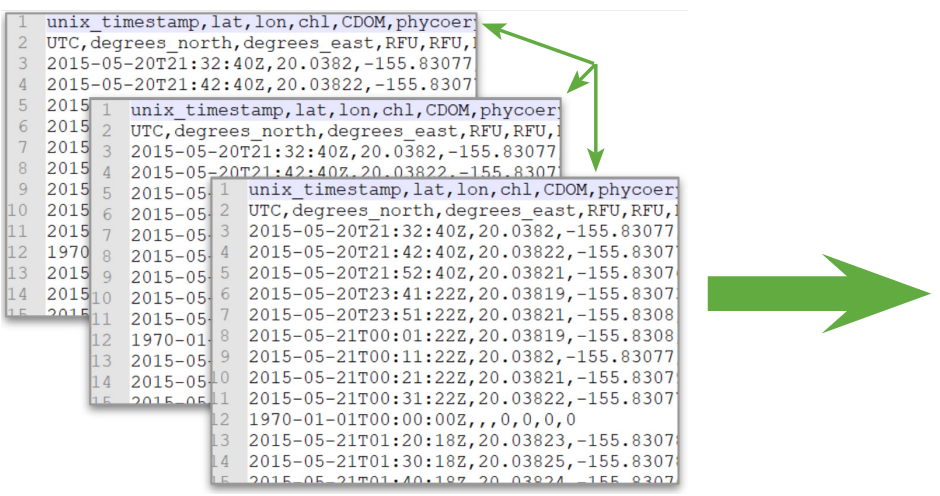

Excel file

\section{After}

Imported into BCO-DMO's data system

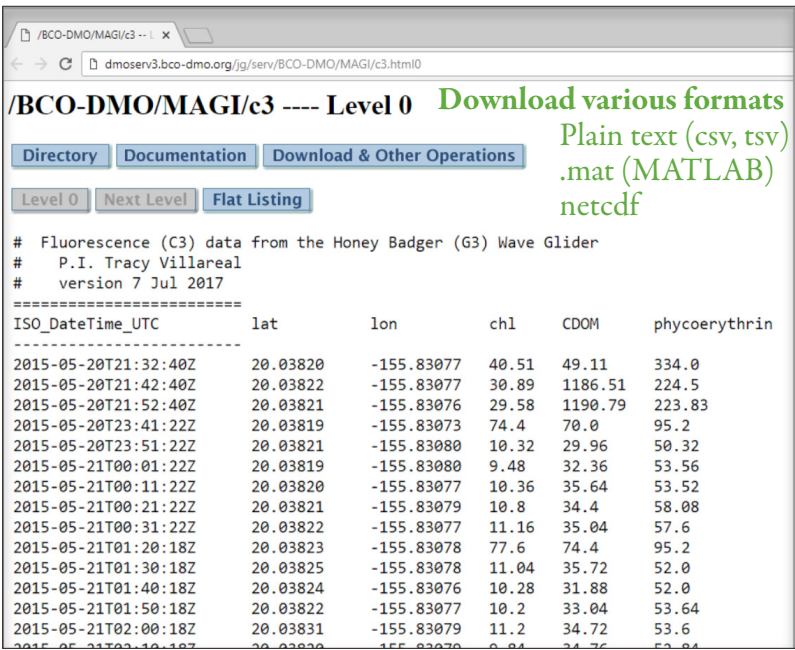

Different data types cannot be combined; these are split into separate datasets.

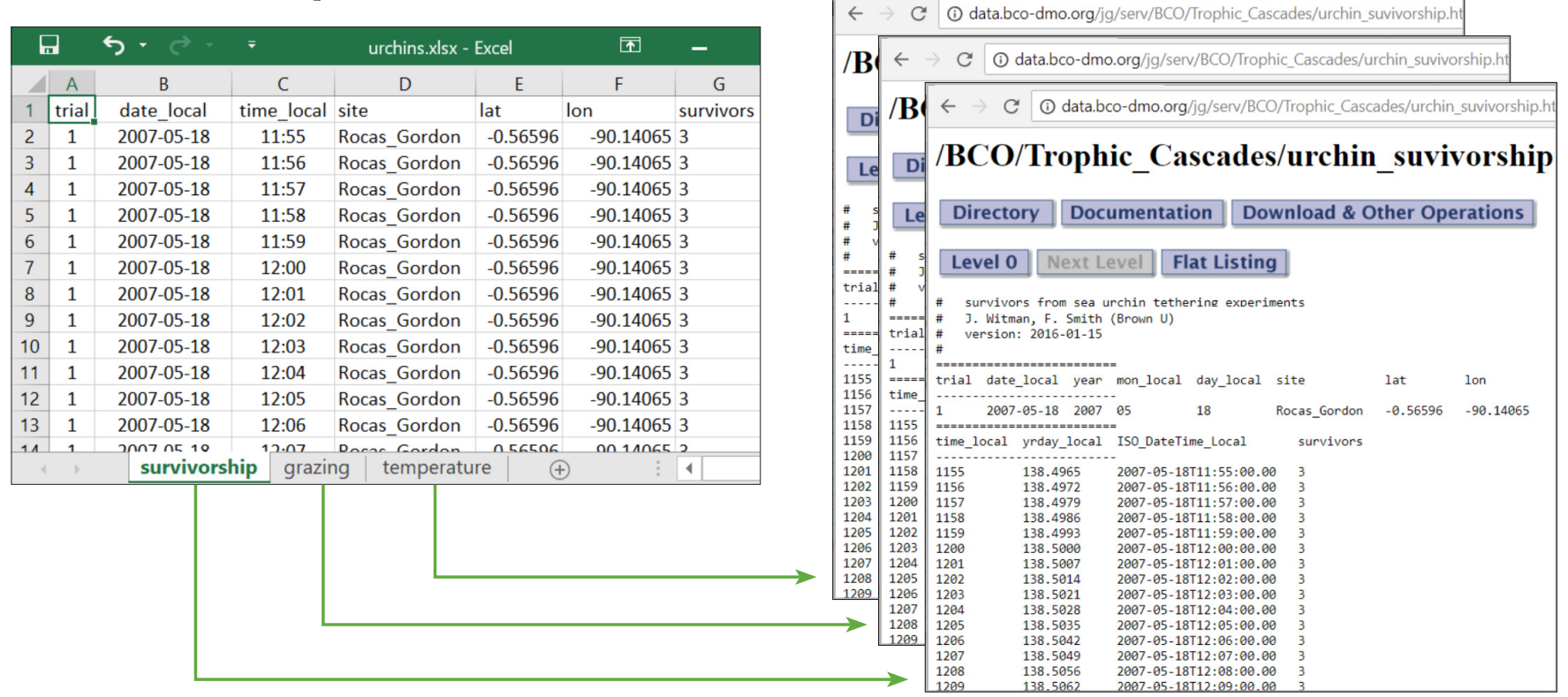




\section{Data Preparation Tips}

\section{General tips:}

- Round your data to the appropriate number of decimal places.

- Make sure all flags and codes are documented in your metadata.

- Submit measured or observed values, not just statistical and calculated values.

\section{Excel files:}

- Remove formatting that will not be preserved when exported as a plain text file (e.g. color, merged cells, plots, etc.)

- Only include one tabular dataset (i.e. table) per Excel sheet.

- Ensure cells contain intended values. Check formula results, references to other sheets, hyperlinks, etc.

\section{Error checking:}

- QA/QC your data before submitting.

- Check species name for correct spelling and use taxonomically accepted names.

\section{Dates \& locations:}

- Document your time and date format including time zone (e.g. UTC, UTC+02, local EST).

- Check for inconsistent date/time formatting.

- In-situ data: include date/time and lat/lon.

- Experimental data: include date/time of experiments if applicable.

\section{Connecting Data and Metadata}

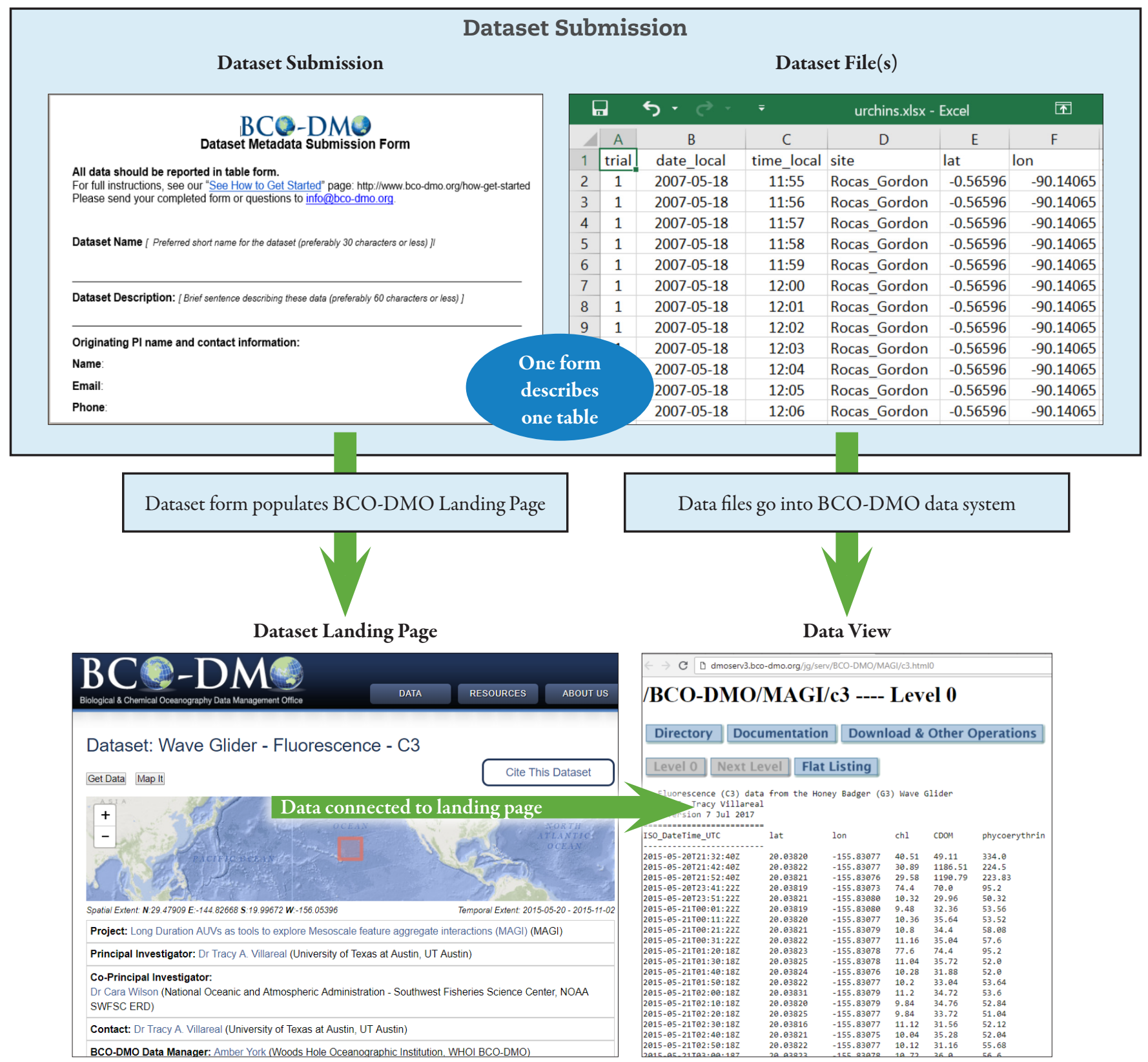




\section{Metadata}

BCO-DMO uses a form to capture important information about your dataset, such as where and how it was collected, analysis methods, and funding sources. This information is known as "metadata". The metadata you provide about your data through the form should be thorough, complete, and publication ready. The contents of your metadata form are directly used to populate the public Dataset Landing Page.

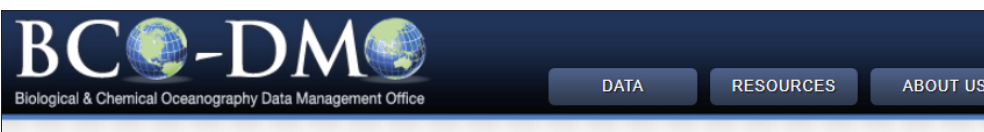

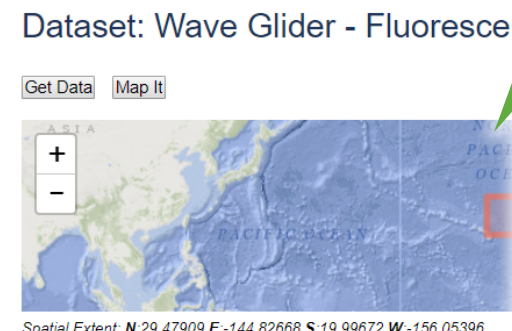

Spatial Extent: N:29.47909 E-144 82668 S 19.99672 W:-156.05396

Project: Long Duration AUVs as tools to explore Mesoscale

\begin{tabular}{l} 
Principal Investigator: Dr Tracy A. Villareal (University of 7 \\
$\begin{array}{l}\text { Co-Principal Investigator: } \\
\text { Dr Cara Wilson (National Oceanic and Atmospheric Adminis } \\
\text { SWFSC ERD) }\end{array}$ \\
\hline Contact: Dr Tracy A. Villareal (University of Texas at Austin
\end{tabular}

Current State: Final no updates expected

Data URL: https://www.bco-dmo.org/dataset/653653/data 〔

\section{Dataset Metadata Form}

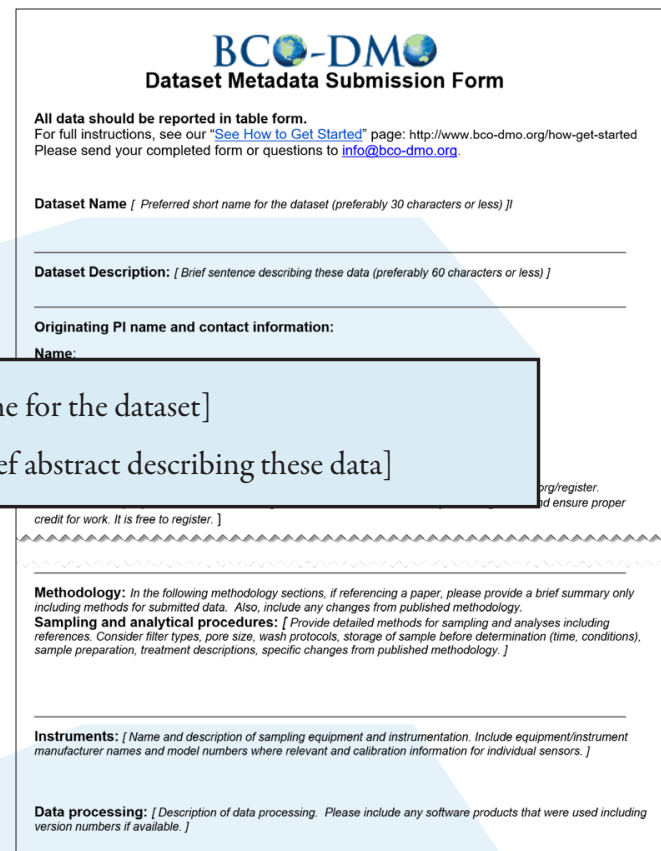

Fluorescence data (C3) collected by the AUV Honey Badger (Wave Glider) in th (MAGI project)

Expand/Collapse All

- Description

This dataset includes chlorophyll, phycoerythrin, and CDOM V2) during a 2015 deployment in the North Pacific Ocea

For more information on project MAGI and a de on of Honey Badger, see: http://oceanview.pfeg.noaa. go

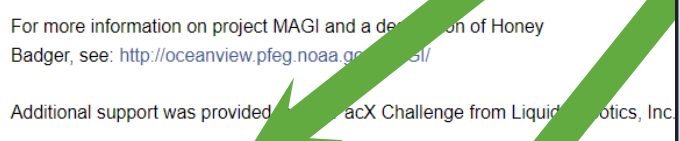

Additional support was provided acX Challenge from Liquid otics, Inc

Acquisition Description

Data were collected at the surface by the the AUV Hon didger (a Wave Glider(R) model SV2 from Liquid Robotics). This deployment in the North Pacific Oce part of Project MAGI. For more details about the Honey Badger and project MAGI please see project pad

http://oceanview.pfeg.noaa.gov/MAGI

Chlorophyll, phycoerythrin, and CDON a were aquired from two float-mounted Turner Designs' $\mathrm{C}^{\mathrm{TM}}$ Submersible Fluorometers. Biofou' o a big issue for any long deployment vehicle, and having two sensors provided the redundancy nee le data quality assurance. No calibration was deemed useful due to the duration of the mission and nature of ions asked

- Processing Description

No calibration was deemed useful due to the duration of the mission and nature of questions asked. Sensors returned only RFU.

BCO-DMO Data Manager Processing Notes:

* added a conventional header with dataset name, PI name, version date

* modified parameter names to conform with BCO-DMO naming conventions

* blank values replaced with no data value 'nd'

* values of "NaN" and "nan" changed to "nd"
Methodology: [In the following methodology sections, if referencing a paper, please provide a brief summary only including methods for submitted data. Also, include any changes from published methodology.]

Sampling and analytical procedures: [ Provide detailed methods for sampling and analyses including references. Consider filter types, pore size, wash protocols, storage of sample before determination (time, conditions), sample preparation, treatment descriptions, specific changes from published methodology.] 
Dataset Metadata Form

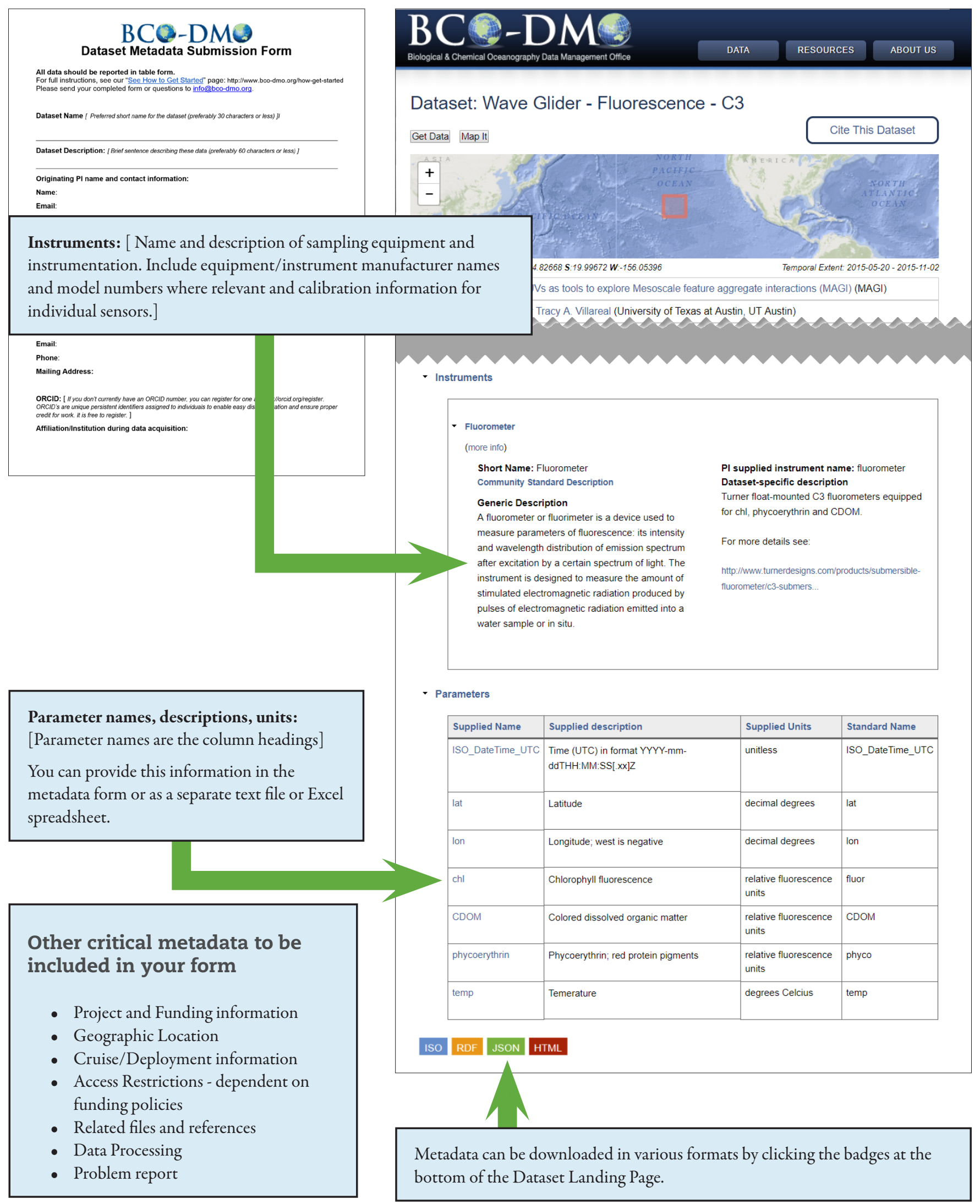




\section{Data Publication}

1 BCO-DMO publishes data and metadata, fostering data discoverability, access, reuse, and attribution.

2 DOIs are generated for every dataset and will be assigned for all submissions. All datasets must be final and validated before a DOI is assigned.
All datasets available at $\mathrm{BCO}-\mathrm{DMO}$ are licensed under a Creative Commons Attribution 4.0 International license, ensuring that each data contributor will receive proper credit.

5 All DOIs are minted for archive by the WHOI Open Access Server (WHOAS), and resolve to WHOAS landing pages.

3 We provide a recommended citation, so that users can properly cite each dataset.

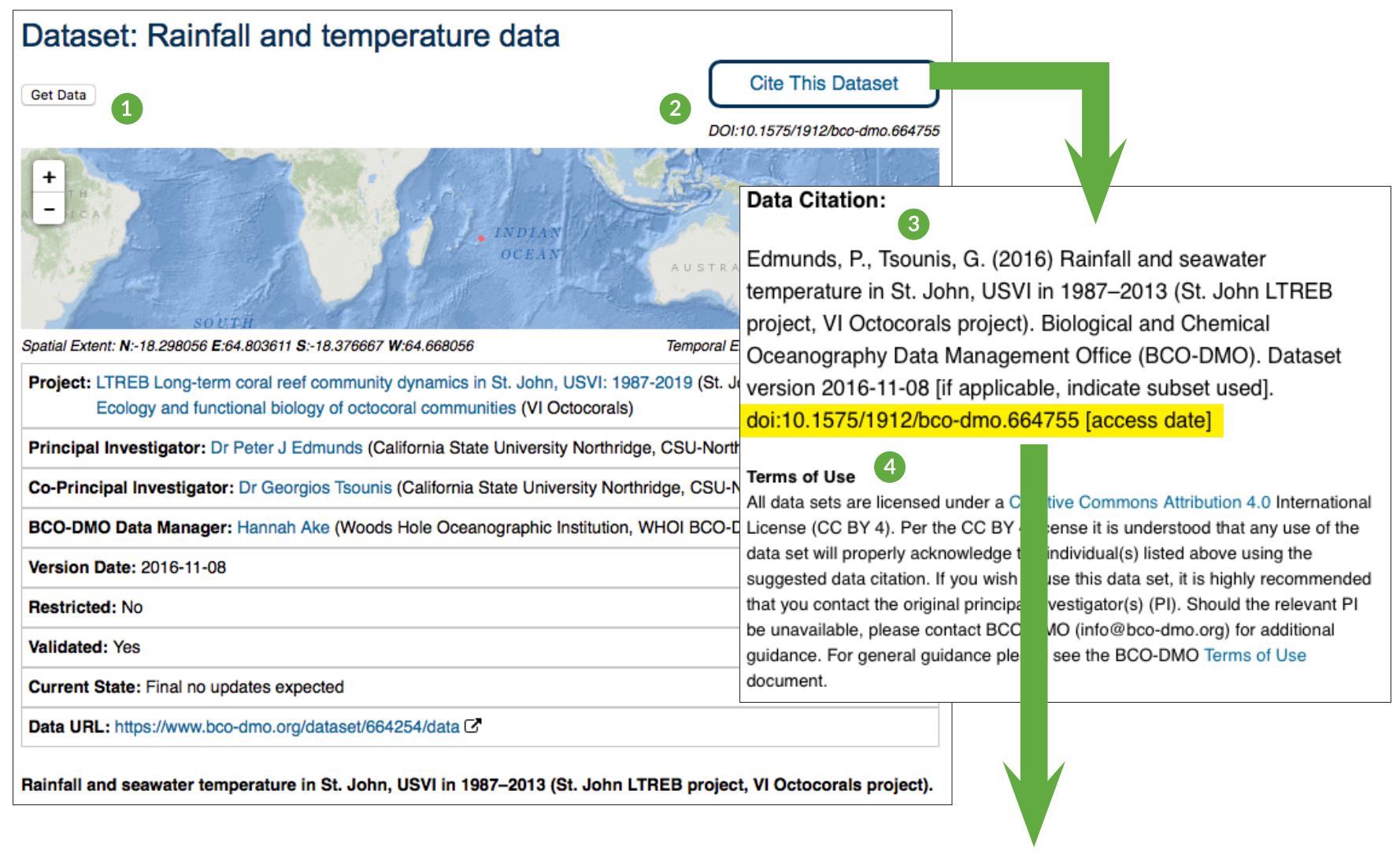

Rainfall and seawater temperature in St. John, USVI in 19872013 (St. John LTREB project, VI Octocorals project).

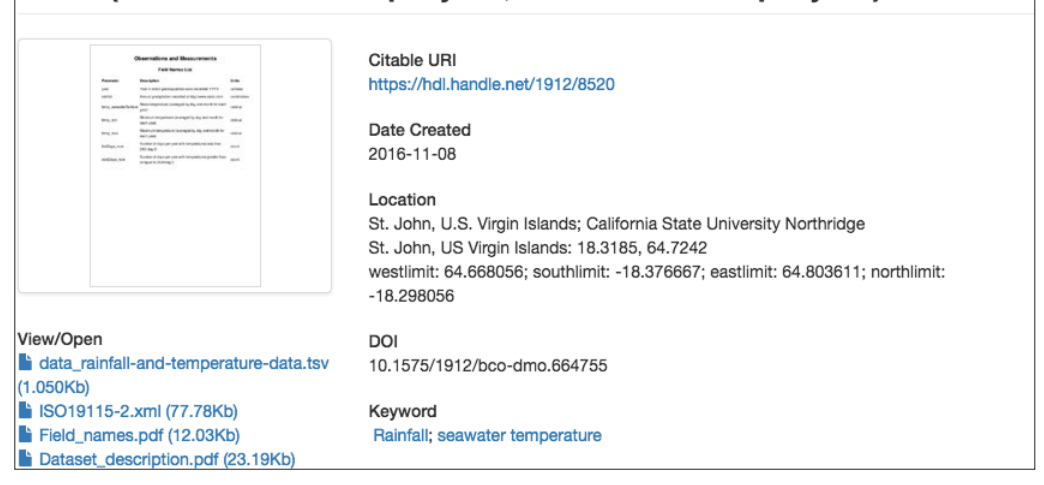




\section{Discovery and Access}

Once data are processed and published online, the BCO-DMO website enables data discovery via text and geospatial search interfaces, making it easy for users to find datasets of choice. Through text-based searches, the database can be searched by cruise, project, person, or any keyword provided in metadata upon submission. Access to data is made possible from the Dataset Landing pages, and data may be subsetted, plotted, and reformatted prior to download. The BCO-DMO database encompasses the full range of oceanographic measurement types from limnological, physical, chemical, biological and/or ecological, and biogeochemical sub-domains.

\section{http://bco-dmo.org/}

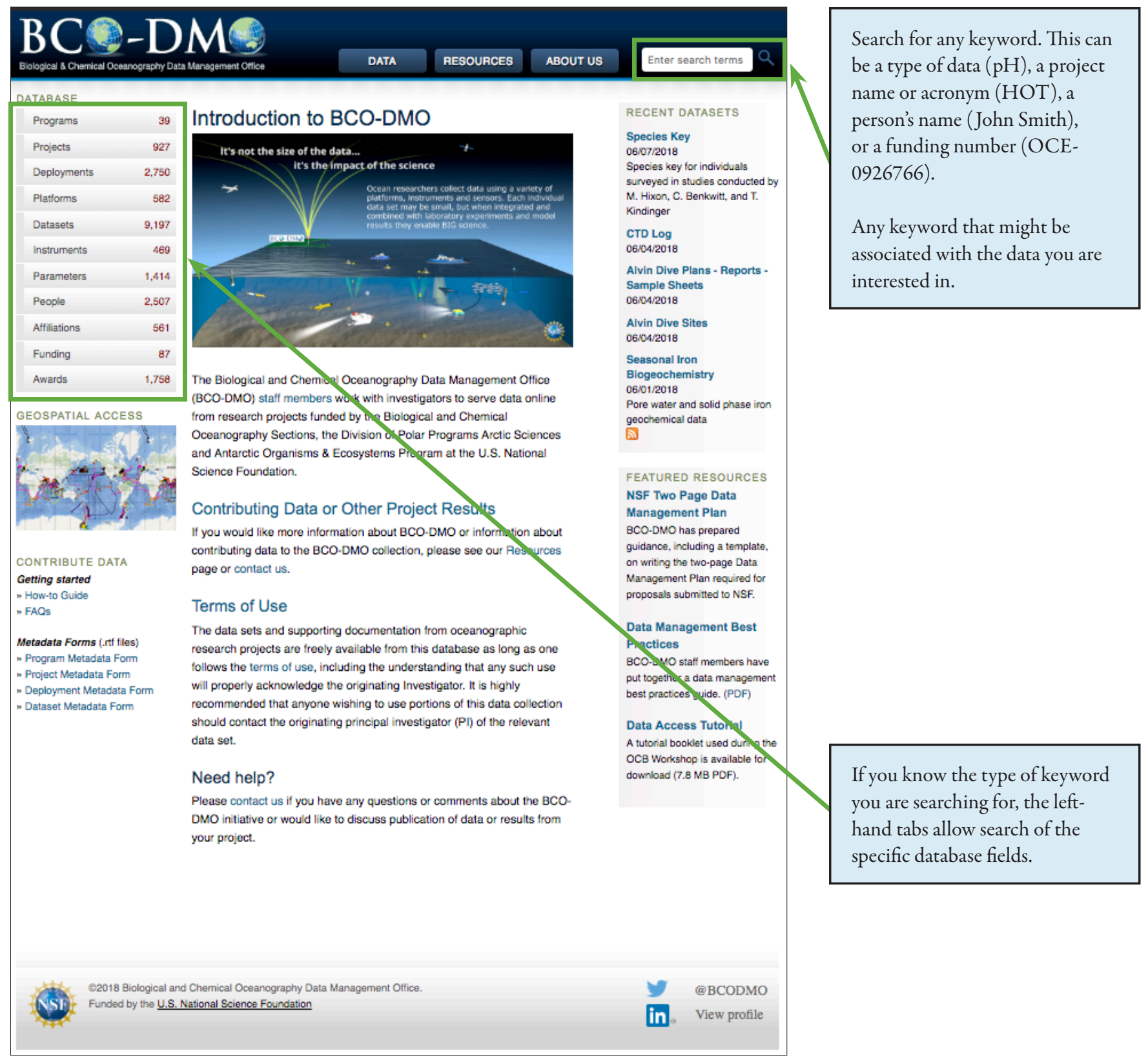

The following example searches for the project HOT (the acronym for Hawaiian Ocean Time-series) and downloads the niskin bottle data. 


\section{Discovery and Access}

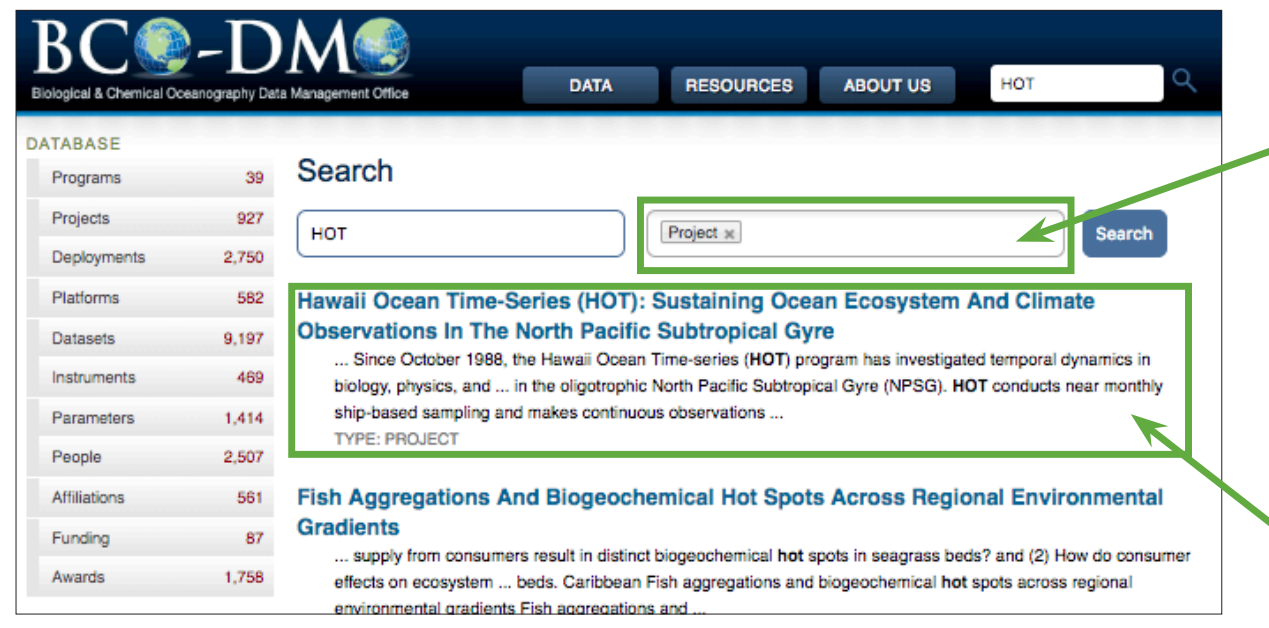

\section{BCQ-DMO}

DATABASE

Programs

Projects

Deployments $\quad 2750$

Plattorms 582

Datasets $\quad 9,197$

(n)

People

Affiliations

Funding

Awards

geOsPATIAL ACCESS

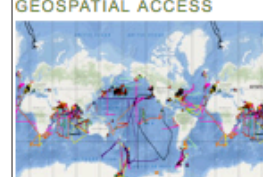

CONTRIBUTE DATA

Getting started

- How-to Guide

-FAQ

Metadata Forms (-rtt files)

- Program Metadata Form

Project Metadata Form

- Deployment Metadata Form

- Dataset Metadata Form
Project: Hawaii Ocean Time-series (HOT): Sustaining ocean

ecosystem and climate observations in the North Pacific

\section{Subtropical Gyre}

Acronym/Short Name: HOT

Data URL: Data [C

Start Date: 1988-07

End Date: 2014-12

Geolocation:

North Pacific Subtropical Gyre; 22 deg 45 min N, 158

$\operatorname{deg} W$

Datasets: 13

Collections: 10

Deployments: 5

Cruises: 4

Platform: 1

Programs:

Ocean Carbon and Biogeochemistry [OCB]

U.S. Joint Global Ocean Flux Study [U.S. JGOFS]

ocean Time-series Sites [Ocean Time-series]

Expand/Collapse AII

- Description

- More Information

- Funding

- Dataset Collections

Additional data for this site are managed by and directly available from the project data site: http://hahana.soest.hawaii.edu/hot/hot-dogs/interlace.htm

\begin{tabular}{|l|l|}
\hline Dataset Short Name & Full Dataset Title \\
\hline $\begin{array}{l}\text { Cruise Track-C- } \\
\text { MORE and HOT } \\
\text { Cruises }\end{array}$ & $\begin{array}{l}\text { Cruise Tracks from RN Kilo Moana, } \mathrm{A} / \mathrm{N} \mathrm{Ka} \text { Kmikai-O-Kanaloa KMO325, KOKO220, } \\
\text { KMO608, KMO627 near Hawaii (22.75 N, 158 W) from 2002-2006 (C-MORE project, HOT } \\
\text { project) }\end{array}$ \\
\hline CTD Profiles & $\begin{array}{l}\text { Two decibar-averaged CTD profiles trom the Hawaii Ocean Time-Series cruises from } \\
1988-2016 \text { (HOT project) }\end{array}$ \\
\hline DNA Extracts & $\begin{array}{l}\text { ONA extracts from the vicinity of Station ALOHA (22.75 N, 158.0 W) just north of Hawaii } \\
\text { from 2007-2015 (C-MORE project, HOT project) }\end{array}$ \\
\hline DNA Time Series & $\begin{array}{l}\text { ONA metagenomic library statistics from HOT cruises from 2007-2009 (C-MORE project, } \\
\text { HOT project) }\end{array}$ \\
\hline $\begin{array}{l}\text { Methane } \\
\text { concentrations at } \\
\text { Station ALOHA }\end{array}$ & $\begin{array}{l}\text { Methane concentrations (depths of 5-175 m) at Station ALOHA collected during Hawail } \\
\text { Ocean Time-Series cruises between 2008 and 2016 (HOT project) }\end{array}$ \\
\hline $\begin{array}{l}\text { Niskin bottle samples } \\
\text { Niskin bottle water samples and CTD measurements from the Hawaii Ocean Time-Series } \\
\text { cruises from 1988-2016 (HOT project) }\end{array}$ \\
\hline $\begin{array}{l}\text { Nitrous oxide } \\
\text { concentrations at } \\
\text { Station ALOHA }\end{array}$ & $\begin{array}{l}\text { Nitrous oxide concentrations (depths of 5-175 m) at Station ALOHA collected during } \\
\text { Hawaii Ocean Time-Series cruises between 2008 and 2016 (HOT project) }\end{array}$ \\
\hline
\end{tabular}

If your search does not return the result of interest, try to filter the search. In this case, we can filter by the type "Project" since we know it's a project in our system.

Clicking on the title will take you to the metadata page for the type of record you select (defined in grey here, as TYPE: PROJECT). There you can see various metadata elements describing the record. This includes individual datasets associated with that record.
The Dataset Collections section of the Project metadata page provides links to datasets associated with this specific project.
The Dataset Short Name link will take you to the Dataset Landing page for that dataset. 


\section{Discovery and Access}

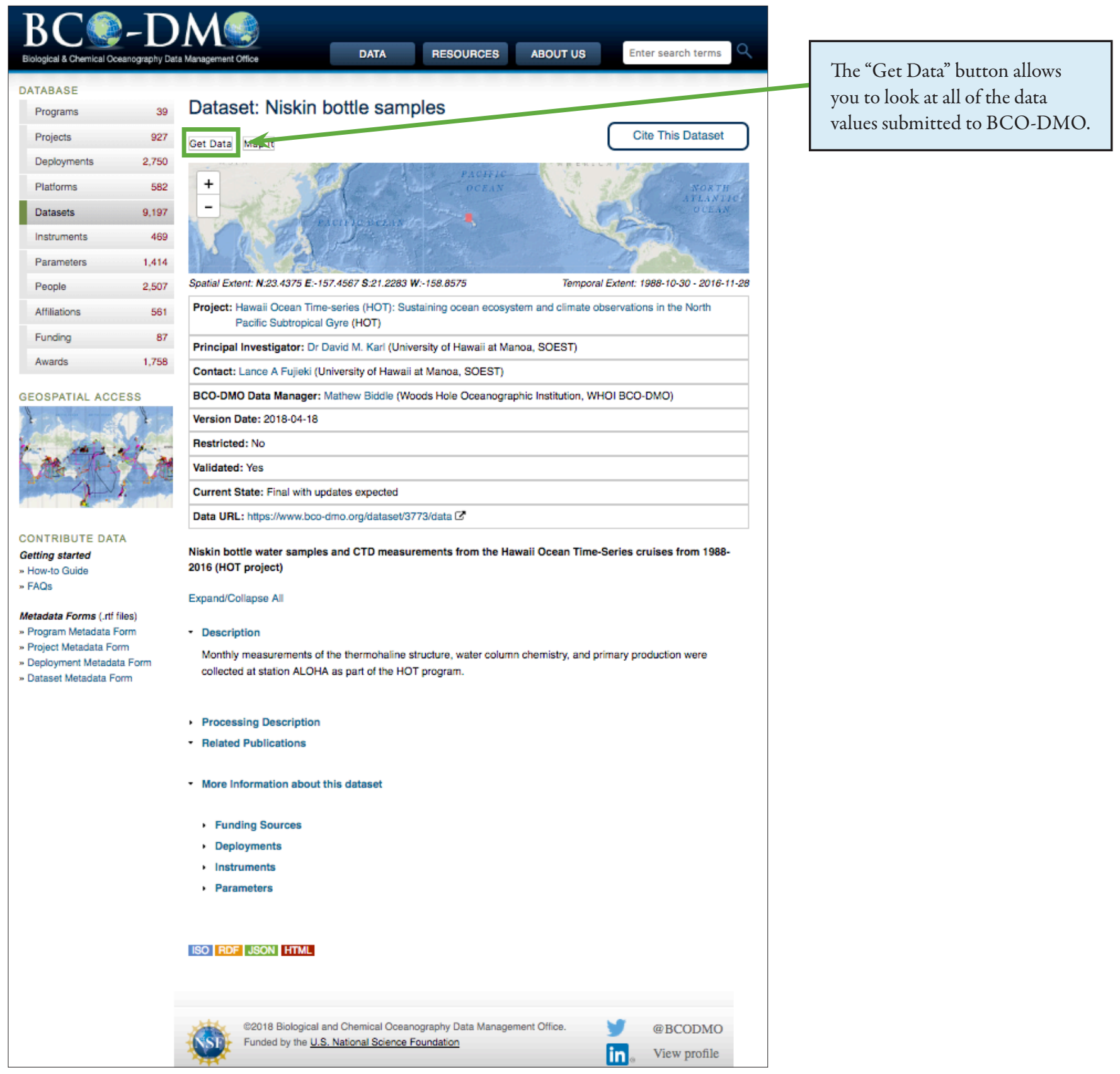




\section{Discovery and Access}

This page displays data values in a hierarchical view (beginning at "Level 0"). Blue text indicates clickable values that expand to uncover more data.

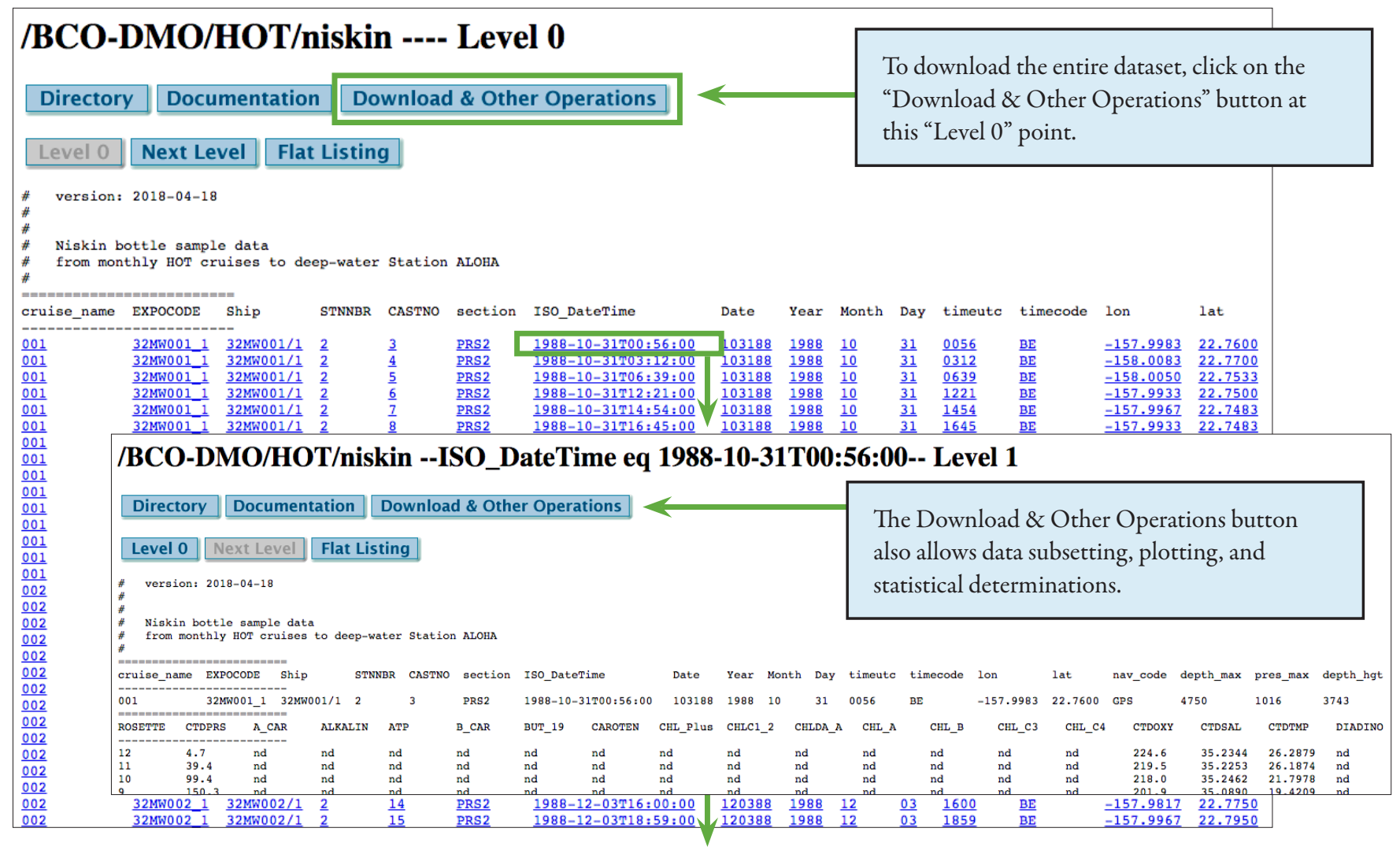

\section{Download and Other Operations Menu}

Current object is: //dmoserv3.bco-dmo.org:80/BCO-DMO/HOT/niskin(ISO_DateTime eq 1988-10-31T00:56:00)

- Listing data

- List at this level

- $\underline{\text { Other data listing formats }}$

- Downloading data

- Matlab file format of all data at this level and further in. (Download will also 'do' matlab) Help

- netcdf file format

- ODV file format Help

- Download utility. Help

This allows direct download of the data.

- Download inquiry/data pickup Help.

- Manipulating data

- Math operations for calculating values from existing parameters. Help.

- Join 2 objects having at least 1 parameter in common. Help.

- Time field splitting Help

- Time conversions

- Statistics

This allows statistical determinations on the data.

- Plotting data - Simple X-Y plot

- Persistent objects

- Directory of available objects

- $\underline{\text { Save/remove current object }}$

- Subsetting data Help

otheropt version $2.2 \mathrm{~h} 9 \mathrm{Mar} 13$

server: production-Apr 17 


\section{Preservation}

Preservation marks a maturity level that allows data to begin the data life cycle again in new research endeavors. BCO-DMO serves as a domain specific, intermediate data repository, and as such does not function as a long-term archive for data preservation. BCO-DMO provides data management support throughout a project award's period of performance which, prepares project output for reuse and reanalysis by the community. Once a project's data and metadata are published online at BCO-DMO, they are then submitted to an appropriate national data center for long-term preservation (e.g., the National Centers for Environmental Information).

\section{FAQ's}

Many Frequently Asked Questions are also addressed on our website at https://www.bco-dmo.org/faq-page. Still have questions? Feel free to contact the office at info@bco-dmo.org and a team member will respond.

\section{Acknowledgements}

The Biological and Chemical Oceanography Data Management Office (BCO-DMO) is funded by the U.S. National Science Foundation award 1435578. The office gratefully acknowledges the Woods Hole Oceanographic Institution's Graphics Department for their support and creativity in website development and outreach materials.

\section{$y$}

\section{Find us on twitter at @BCODMO}

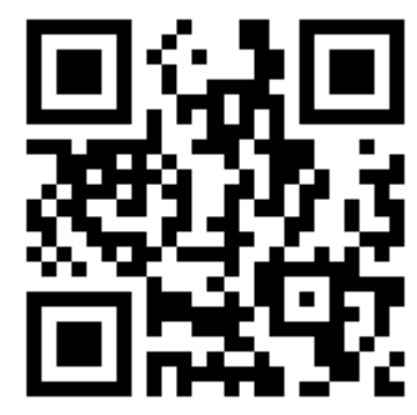

